\title{
Wälder in der Zeitmaschine - Möglichkeiten und Grenzen der Paläoökologie
}

\author{
Christoph Schwörer \\ Brigitta Ammann \\ Marco Conedera \\ Willy Tinner
}

\author{
Pflanzenwissenschaften und Oeschger-Zentrum für Klimaforschung, Universität Bern (CH)* \\ Pflanzenwissenschaften, Universität Bern $(\mathrm{CH})$ \\ Eidgenössische Forschungsanstalt für Wald, Schnee und Landschaft $(\mathrm{CH})$ \\ Pflanzenwissenschaften und Oeschger-Zentrum für Klimaforschung, Universität Bern $(\mathrm{CH})$
}

\begin{abstract}
Wälder in der Zeitmaschine - Möglichkeiten und Grenzen der Paläoökologie
Die Paläoökologie erlaubt es, ökologische Prozesse, die sich über sehr lange Zeiträume abspielen, zu rekonstruieren - zum Beispiel die Vegetationsdynamik seit der letzten Eiszeit oder die Veränderung der Waldzusammensetzung im Zuge der menschlichen Besiedelung. Sie nutzt dazu Pflanzenreste wie Pollen, Sporen, Blätter, Samen und Holzkohle, die unter Luftabschluss in Seesedimenten und Mooren über Jahrtausende konserviert worden sind. Im Artikel werden die Prinzipien, die Grenzen und das Potenzial der Paläoökologie aufgezeigt. Dabei wird deutlich, dass diese Disziplin heute weit mehr als eine beschreibende Wissenschaft ist. Dank quantitativen Methoden wird sie mittlerweile auch dazu verwendet, um ökologische Hypothesen zu testen und kausale Zusammenhänge herzustellen. Die Paläoökologie kann so dazu beitragen, die Anpassungsfähigkeit oder Resilienz von Vegetationsgemeinschaften gegenüber äusseren Störungen abzuschätzen, und liefert damit wichtige Hinweise zur Reaktion unserer Wälder auf den vergangenen, gegenwärtigen und künftigen Klimawandel.
\end{abstract}

Keywords: long-term vegetation dynamics, climate change, human impact, paleoecological methods doi: $10.3188 /$ szf. 2019.0117

*Paläoökologie, Altenbergrain 21, CH-3013 Bern, E-Mail christoph.schwoerer@ips.unibe.ch
Ö kologische Prozesse spielen sich in verschiedensten Zeiträumen ab (Abbildung 1). Am unteren Ende der Skala stehen Extremereignisse, die sich über Stunden, Tage oder Wochen abspielen, zum Beispiel Windwurf, Waldbrände, Krankheiten oder Hitzeperioden. Am oberen Ende der Skala stehen Prozesse, die sich über Jahrtausende oder länger hinziehen, beispielsweise die Ausbreitung von Pflanzen nach der letzten Eiszeit oder die Bildung neuer Arten. Heutzutage befasst sich die Ökologie vor allem mit Prozessen, die sich über wenige Monate bis Jahre abspielen und mit Experimenten überprüft werden können. Bereits die Reaktion auf kurzfristige Ereignisse wie die Sukzession nach einem Waldbrand kann jedoch mehrere Jahrzehnte bis Jahrhunderte dauern und ist mit den Methoden der experimentellen Ökologie nur schwer zu erfassen. Noch schwieriger wird es bei Prozessen, die sich über Jahrtausende abspielen wie die Langzeitdynamik von Baumpopulationen oder die Veränderung der Artenzusammensetzung unserer Wälder im Zuge der menschlichen Landnutzung. Um solche Fragestellungen zu ergründen, bietet die Paläoökologie wertvolle Hilfe. Diese Disziplin bewegt sich im Über- lappungsbereich von Botanik, Vegetationsökologie, Paläoklimatologie, Archäologie und Quartärgeologie. Sie nutzt die in natürlichen Archiven gespeicherten Informationen, um Veränderungen über lange Zeiträume zu untersuchen (Abbildung 1).

Dank dieser langfristigen Betrachtungsweise sind die aus der Paläoökologie gewonnenen Informationen gerade bei der aktuellen Diskussion über die Zukunft unserer Wälder sowie die Wettbewerbsund Anpassungsfähigkeit der einzelnen Pflanzenarten unter veränderten Klimabedingungen sehr wichtig. Die Paläoökologie ermöglicht es, die Reaktion der Vegetation auf vergangene Klimaänderungen zu untersuchen und daraus Schlüsse für die Zukunft zu ziehen. Zu solchen analogen Klimaänderungen gehört zum Beispiel der Übergang vom Ende der letzten Eiszeit zur jetzigen Warmzeit (dem Holozän) vor 11700 Jahren, als die Sommertemperaturen innerhalb weniger Jahrzehnte um zwei bis vier Grad Celsius angestiegen sind (Heiri et al 2014). Ein solcher Temperaturanstieg entspricht etwa den Klimaprognosen für das Ende dieses Jahrhunderts (CH 2018). In diesem Beitrag stellen wir die Prinzipien, die Grenzen und das Potenzial der Paläoökologie vor 


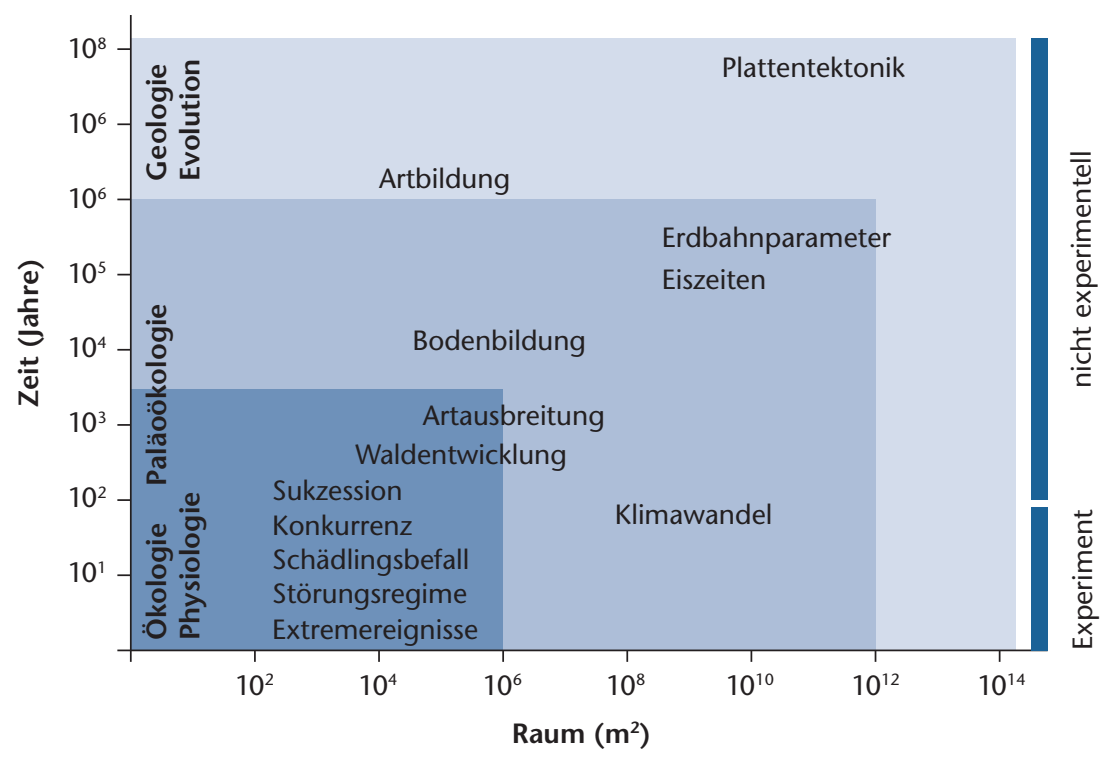

Abb 1 Raum- und Zeitskalen von ökologischen Prozessen (hier logarithmisch dargestellt) sowie Methoden und Disziplinen für ihre Untersuchung.

und legen dabei ein besonderes Augenmerk auf die Anwendungsmöglichkeiten im forstlichen Bereich.

\section{Grundsätze der Paläoökologie}

Der paläoökologische Ansatz stützt sich auf folgende wichtige Grundprinzipien: 1) die Existenz von geeigneten natürlichen Archiven mit intakten organischen Resten, 2) die unterschiedliche Morphologie der organischen Reste, die einen Rückschluss auf die entsprechende Familie, Gattung oder gar Art erlaubt, 3) die Datierung der Archive, die den Pflanzenresten ein bestimmtes Alter zuweist, 4) die Kalibrierung und die statistische Auswertung der Ergebnisse, die es erlauben, allfällige Kausaleffekte oder Rückkoppelungen in der Entwicklung der Pflanzen-

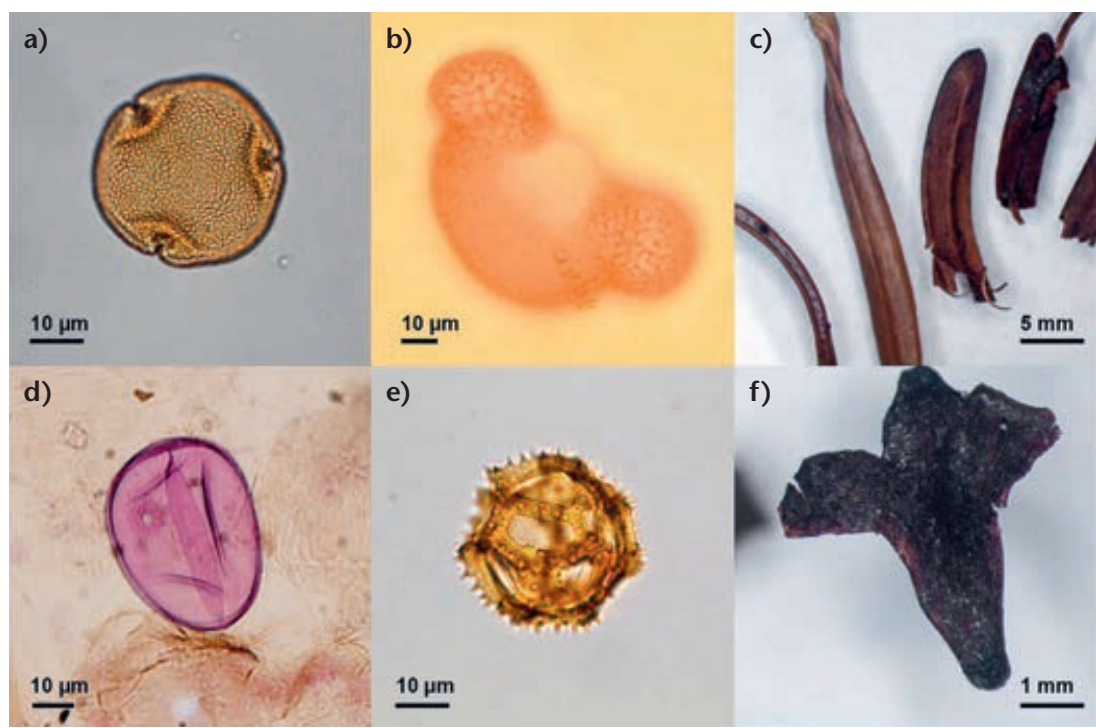

Abb 2 Mikro- und Makrofossilien verschiedener Pflanzenarten. a) Pollen der Linde (Tilia sp.), b) Pollen der Kiefer (Pinus-sylvestris-Typ), c) Nadelreste der Weisstanne (Abies alba), d) Pollen von Roggen (Secale), e) Pollen eines Zungenblütlers (Cichorioidae, Asteraceae), f) Fruchtschuppe der Birke (Betula sp.). Zur Pollenmorphologie siehe Beug (2004). reste zu belegen, und 5) die Verbindung unterschiedlicher und voneinander unabhängiger Methoden, um zuverlässige Rückschlüsse auf die vergangenen Umweltbedingungen und -prozesse zu ziehen.

\section{Wahl geeigneter Untersuchungsstandorte}

Als natürliche Archive werden Standorte bezeichnet, die Umweltinformationen über Jahrhunderte oder Jahrtausende aufzeichnen. Für die Paläoökologie sind dies insbesondere Seen und Moore, da hier Pflanzenreste unter Luftabschluss über Jahrtausende im Sediment erhalten bleiben. Voraussetzung für erfolgreiche Analysen ist ein sauberer, ungestörter Bohrkern. Dies bedingt, dass der See in der Vergangenheit nie ausgetrocknet ist oder Hangrutschungen erfahren hat und keine grösseren Zuflüsse besitzt, die grosse Geschiebemengen ablagern können. Aus technischen Gründen darf der See auch nicht zu tief sein. Ideal sind Seen mit einer Tiefe von 3 bis 60 Metern und einer Grösse von 0.1 bis 1000 Hektaren. Auch Moore eignen sich - nicht zuletzt, da viele aus der Verlandung von Seen hervorgegangen sind. Durch die Wassersättigung und das saure Milieu bleiben Pflanzenreste auch im Torfkörper über lange Zeiträume erhalten.

\section{Morphologie der Pflanzenreste}

Bei den Pflanzenresten unterscheidet man in der Paläoökologie zwischen Mikrofossilien wie Pollen, Sporen und anderen leicht erkennbaren Zellen (z.B. Spaltöffnungen) sowie Makrofossilien wie Samen, Holz oder Blätter (Abbildung 2). Dank dem Formenreichtum können diese biotischen Überreste unter dem Binokular oder Mikroskop auf Familie, Gattung oder Art bestimmt werden. Bei gut erhaltenen Makrofossilien ist eine Bestimmung in den meisten Fällen bis auf Artebene möglich. Allerdings sind grössere Pflanzenreste in den Archiven oft nur in tiefen Konzentrationen vorhanden. Äusserst zahlreich kommen dagegen Mikrofossilien vor. Während Mikrofossilien der meisten europäischen Waldbäume bis auf die Art bestimmt werden können, gelingt das bei den meisten anderen Artengruppen, besonders bei Kräutern, oft nur bis zur Gattung oder Familie. Für die Pollenanalyse werden Sedimentproben von einem Kubikzentimeter chemisch und physikalisch behandelt, um Verunreinigungen zu entfernen, und dann unter dem Mikroskop bei 400- bis 1000-facher Vergrösserung bestimmt und gezählt. Das Vorkommen und die Häufigkeit verschiedener Pollentaxa erlaubt nun, Rückschlüsse auf die vergangene Vegetation zu ziehen (Lang 1994).

Farn- und Pilzsporen können gelegentlich auch von Interesse sein, zum Beispiel wurde von der Häufigkeit von Pilzarten, die Tierkot besiedeln, auf die Häufigkeit von Mammuts und jungsteinzeitlichen Haustieren geschlossen (z.B. Davis \& Shafer 2006, Hafner \& Schwörer 2018). Ebenfalls von Inte- 


\begin{tabular}{|c|c|c|}
\hline Proxy & Umweltfaktor & Referenz \\
\hline $\begin{array}{l}\text { Mikrofossilien } \\
\text { (z.B. Pollen, Sporen) }\end{array}$ & $\begin{array}{l}\text { Regionale Vegetation, Tempe- } \\
\text { ratur, menschlicher Einfluss }\end{array}$ & Smol et al (2001a) \\
\hline $\begin{array}{l}\text { Makrofossilien } \\
\text { (z.B. Samen, Holz, } \\
\text { Blätter) }\end{array}$ & $\begin{array}{l}\text { Lokale Vegetation, menschli- } \\
\text { cher Einfluss }\end{array}$ & Smol et al (2001a) \\
\hline Holzkohle & $\begin{array}{l}\text { Waldbrände, menschlicher } \\
\text { Einfluss }\end{array}$ & $\begin{array}{l}\text { Conedera \& Tinner (2010), } \\
\text { Smol et al (2001a) }\end{array}$ \\
\hline $\begin{array}{l}\text { Anteil an organischem } \\
\text { Material im Sediment }\end{array}$ & Primärproduktion im See & $\begin{array}{l}\text { Heiri et al (2001), } \\
\text { Last \& Smol (2001a) }\end{array}$ \\
\hline Pigmentanalyse & $\begin{array}{l}\text { Primärproduktion im See, } \\
\text { Nährstoffeintrag }\end{array}$ & Smol et al (2001a) \\
\hline $\begin{array}{l}\text { Kieselalgen } \\
\text { (Diatomeen) }\end{array}$ & $\begin{array}{l}\mathrm{pH} \text {, Nährstoffe, Temperatur, } \\
\text { Salinität }\end{array}$ & Smol et al (2001a) \\
\hline $\begin{array}{l}\text { Zuckmückenlarven } \\
\text { (Chironomiden) }\end{array}$ & $\begin{array}{l}\text { Temperatur, Sauerstoff- } \\
\text { verfügbarkeit, Nährstoffe }\end{array}$ & $\begin{array}{l}\text { Eggermont \& Heiri (2012), } \\
\text { Smol et al (2001b) }\end{array}$ \\
\hline Elementanalyse (XRF) & Redoxverhältnisse, Erosion & Last \& Smol (2001b) \\
\hline $\begin{array}{l}\text { Magnetische } \\
\text { Suszeptibilität }\end{array}$ & Erosion & Last \& Smol (2001b) \\
\hline aDNA & $\begin{array}{l}\text { Artvorkommen, genetische } \\
\text { Diversität }\end{array}$ & $\begin{array}{l}\text { Birks \& Birks (2016), } \\
\text { Gugerli et al (2005) }\end{array}$ \\
\hline Isotope & $\begin{array}{l}\text { Temperatur, Hydrologie, } \\
\text { Methan, Nährstoffkreislauf }\end{array}$ & Last \& Smol (2001a) \\
\hline Korngrösse & Erosion, Hydrologie & Last \& Smol (2001a) \\
\hline Seespiegel & Hydrologie, Klima & Magny (2004) \\
\hline
\end{tabular}

Tab 1 Beispiele von Proxies und daraus abgeleitete Umweltfaktoren, die aus Seesedimenten rekonstruiert werden können.

resse sind das Vorkommen und die Häufigkeit von Holzkohlepartikeln, da damit das Waldbrandgeschehen rekonstruiert werden kann (Conedera \& Tinner 2010).

\section{Datierung der Sedimente}

Wenn organisches Material vorhanden ist, kann dessen Alter mit der Radiokarbonmethode bis maximal 50000 Jahre vor heute bestimmt werden. Diese Methode der Altersbestimmung enthält jedoch immer eine gewisse Unsicherheit durch den vorhandenen Messfehler und die Kalibrierung der Rohdaten. Das Alter einer Probe kann deshalb meist nur auf 50 bis 500 Jahre genau angegeben werden. Für präzisere Altersbestimmungen sind Paläoökologen auf jährlich geschichtete Sedimente angewiesen, die sogenannten Warven, die ähnlich wie die Jahrringe der Bäume eine saisonale Abfolge von unterschiedlich gefärbten Sedimenten aufweisen. Für Radiokarbondatierungen sollten nur schnell abbaubare, eindeutig terrestrische Pflanzenreste verwendet werden (z.B. Nadeln, Blätter, Samen oder kleine Zweige). Aquatische Pflanzenteile und Seesediment können je nach Geologie des Sees einen gewissen Anteil an anorganischem oder mit ${ }^{14} \mathrm{C}$ abgereichertem Kohlenstoff enthalten, der das Alter der Probe um Hunderte bis Tausende von Jahren verfälschen kann (Finsiger et al 2018). Da Radiokarbondatierungen kostspielig und aufwendig sind, werden meist nur einige Proben aus unterschiedlichen Tiefen datiert und dann zwischen den Proben interpoliert. Die Stratigrafie eines Bohrkerns aus einem Moor oder einem See kann so in eine Zeitachse umgewandelt werden.

\section{Statistische Auswertung der Daten}

Eine Reihe statistischer Methoden kann eingesetzt werden, um die paläoökologischen Daten quantitativ auszuwerten. Erwähnenswert ist hier besonders die Gradientenanalyse, die es erlaubt, multivariate Datensätze in wenigen Dimensionen darzustellen und so die wichtigsten Einflussfaktoren zu bestimmen (Birks et al 2012). Falls auch noch Daten zu verschiedenen Umweltfaktoren zur Verfügung stehen, ist es weiter möglich, quantitativ zu bestimmen (z.B. über Kreuzkorrelationen), wie gross der Einfluss der einzelnen Faktoren auf die Vegetation ist (Schwörer et al 2015, Tinner et al 1999).

\section{Multiproxy-Ansatz}

Um ein vollständiges Abbild vergangener Umweltbedingungen zu erhalten, ist es von Vorteil, am selben Sedimentkern verschiedene Indikatoren («Proxies») zu messen. So kann durch die Analyse von Mikro- und Makrofossilien die regionale und lokale Vegetation rekonstruiert werden. Da die Autökologie der meisten Pflanzenarten Europas gut untersucht ist (Ellenberg \& Leuschner 2010), ist die Versuchung gross, Veränderungen in der Vegetationszusammensetzung durch klimatische Ereignisse zu erklären. Dies kann jedoch schnell zu Zirkelschlüssen führen, wenn dann die Vegetationsänderung wiederum als Beleg für die Klimaänderung angegeben wird. Weitaus eleganter ist es, von der Vegetation unabhängige Proxies für das Klima zu verwenden, zum Beispiel Sauerstoffisotope oder die Artenzusammensetzung von Zuckmückenlarven oder Kieselalgen. Zudem ist es für quantitative Analysen unabdingbar, dass voneinander unabhängige Proxies miteinander verglichen werden. Proxies, die oft in der Paläoökologie, der Paläolimnologie und der Quartärgeologie verwendet werden, sind in Tabelle 1 aufgeführt.

\section{Interpretation und Analyse paläoökologischer Daten}

\section{Präsentation paläoökologischer Daten}

Schon vor hundert Jahren stellten Pioniere der Paläoökologie wie Lennart von Post fest, dass die Pollenverteilung im Sediment nicht exakt der Artenverteilung in der Umgebung eines Sees entspricht. Zudem schwankt die Pollenkonzentration im Sedimentkern stark. Um dieses Problem zu umgehen, werden die Pollenfunde in den Diagrammen, deren y-Achse der Kerntiefe oder dem Alter entspricht, 


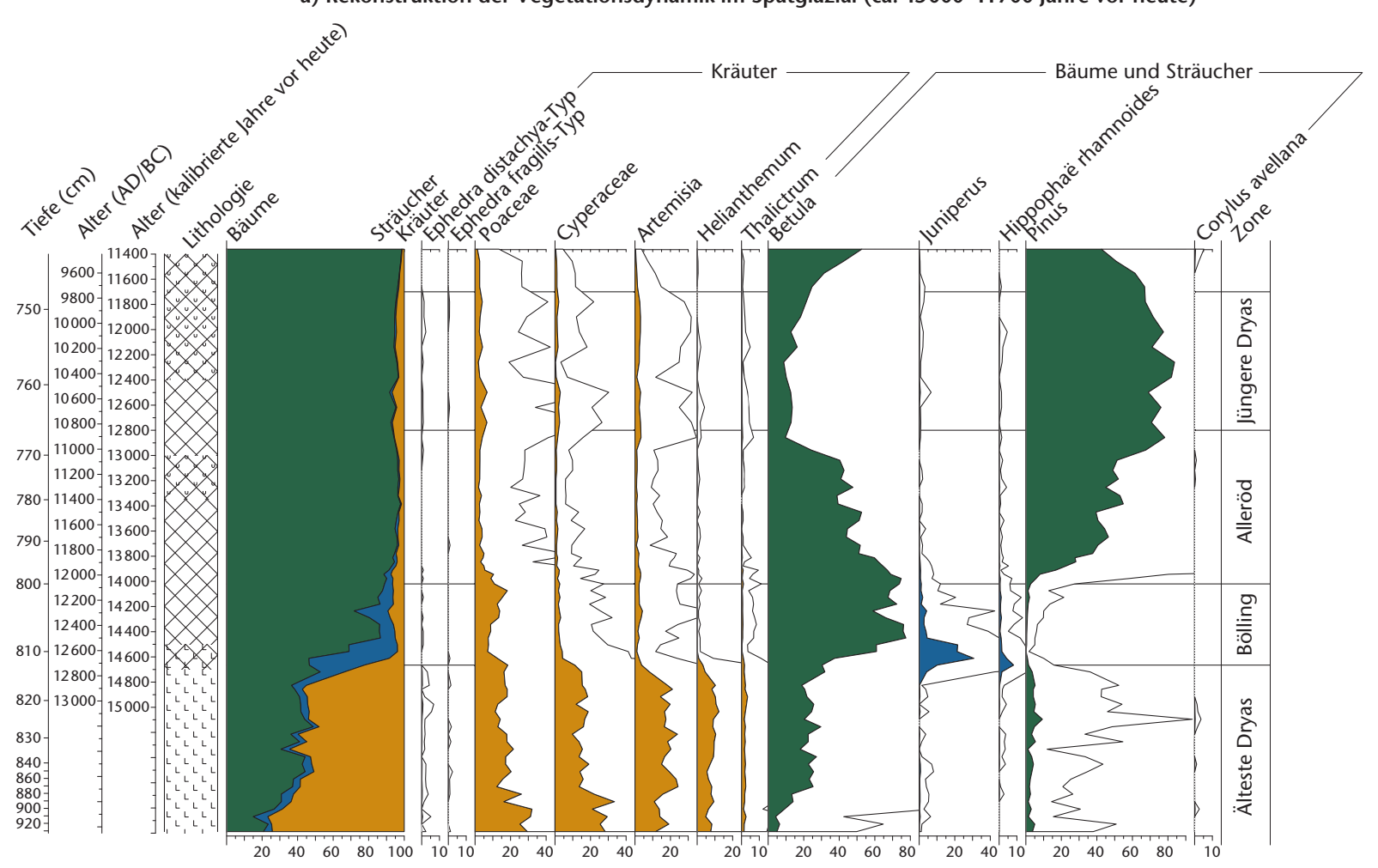

b) Rekonstruktion der Vegetationsdynamik im Holozän (11700 Jahre vor heute bis in die Gegenwart)

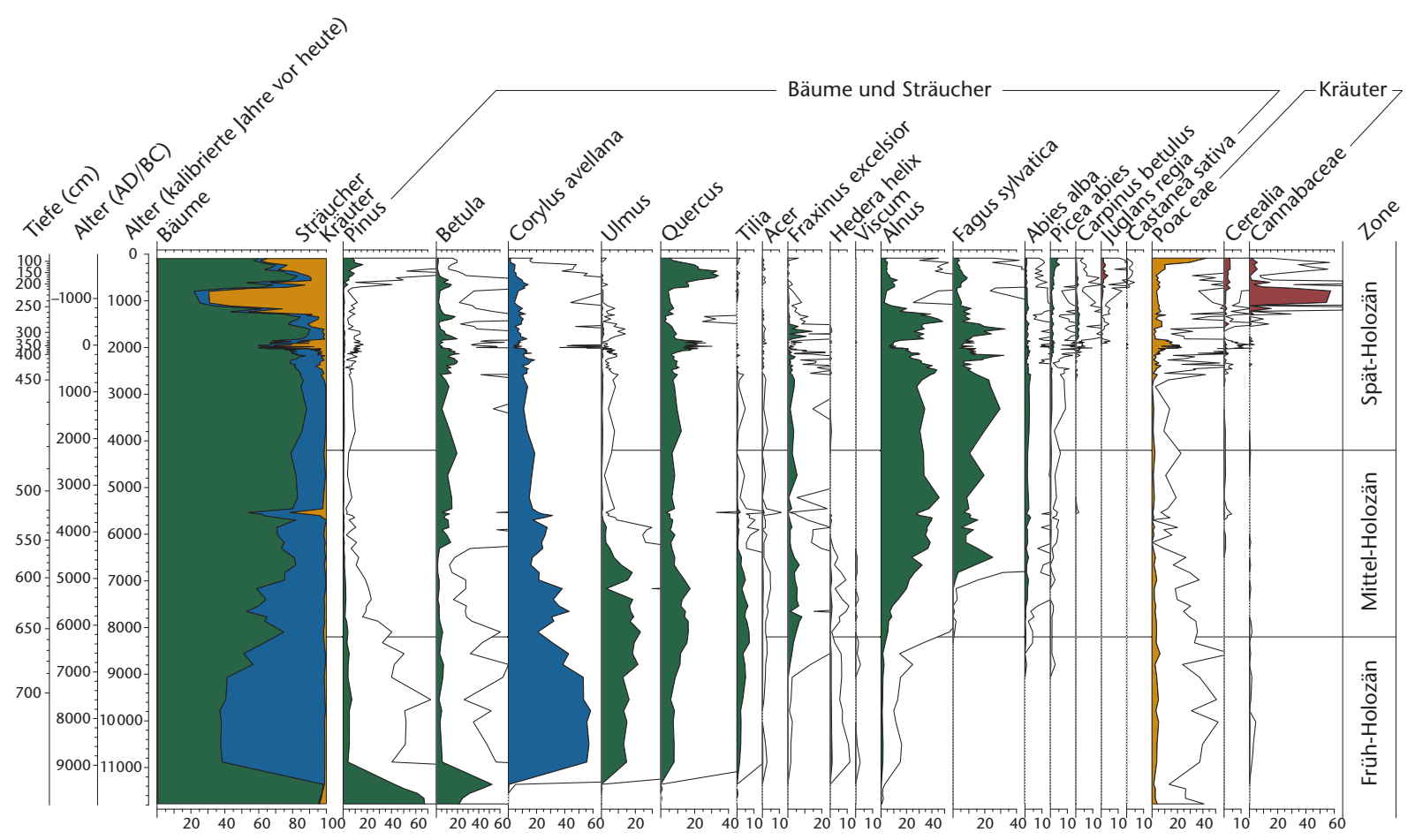

$\square$ Bäume $\square$ Sträucher $\square$ Kräuter $\square$ Landwirtschaftliche Nutzpflanzen $\quad$ Gyttja 國 Seekreide 四 Silt

Abb 3 Pollendiagramme des Lobsigensees im Schweizer Mittelland. Die aus dem Sediment extrahierten und analysierten Pollentypen sind als Prozentwerte der Pollensumme der Landpflanzen dargestellt. Weisse Kurven entsprechen einer 10fachen Überhöhung, um tiefe Werte darstellen zu können.

als Prozente der Gesamtsumme dargestellt (Abbildung 3). Dabei wird meist der Pollen aquatischer Pflanzen aus der Pollensumme ausgeschlossen, da der Fokus jeweils auf der Rekonstruktion der terrestrischen Vegetation (z.B. der Wälder) liegt. Da die Werte einzelner Arten teilweise sehr gering sind oder stark schwanken, werden die Pollenkurven zusätz- lich grafisch überhöht dargestellt (Abbildung 3). Ausführliche Pollendiagramme sind wegen der vielen Taxa, die gezeigt werden, oft nicht einfach zu lesen. Hilfreich ist aber jeweils das Hauptdiagramm, in dem traditionellerweise die Pollensummen der Bäume, der Sträucher und der Kräuter zusammengefasst sind und das die wichtigsten Vegetationsänderungen 
über die Zeit wiedergibt (Abbildung 3). Pollendaten und Holzkohlepartikel werden oft auch als Influx (Anzahl Partikel pro Fläche und Jahr) angegeben, um zu überprüfen, ob Änderungen in den Pollenprozenten tatsächlich Vegetationsänderungen widerspiegeln oder bloss durch die geschlossene Pollensumme (100\%) bedingt sind. Um Influxwerte verwenden zu können, ist eine robuste Chronologie unabdingbar, da die Berechnung des Polleninfluxes direkt vom verwendeten Alters-Tiefen-Modell abhängt.

\section{Kalibrierung der Daten}

Pollendiagramme können keine direkten Informationen auf die Vegetationszusammensetzung liefern, da die Pollenproduktion und -deposition je nach Art und Standort sehr unterschiedlich ist (z.B. produzieren windblütige Arten viel mehr Pollen als insektenbestäubte). Starke Pollenproduzenten wie Pinus sylvestris können auch weit ausserhalb ihres Areals beträchtliche Pollenprozente aufweisen. Die Pollenproduktivität einzelner Baumarten wird primär mit dem Vergleich der Anzahl Pollen in Oberflächenproben (z.B. von Moospolstern, Pollenfallen oder Oberflächensedimenten) und der Häufigkeit der jeweiligen Art in der Umgebung ermittelt (z.B. Welten 1950, van der Knaap et al 2010). Als besonders wichtig ist zudem das sogenannte Polleneinzugsgebiet, das von der Seegrösse abhängt: Grössere Seen wie der Bodensee haben ein deutlich grösseres Einzugsgebiet als kleinere. Bei Seen mit einer Oberfläche von wenigen Hektaren stammt der Grossteil der im Sediment enthaltenen Pollen aus der näheren Umgebung $(<10 \mathrm{~km})$. Bei grösseren Seen nimmt der Pollenanteil, der über grössere Distanzen transportiert wird, zu (Conedera et al 2006, Sugita 2007a, $2007 b)$. So kommt es beispielsweise oft vor, dass Pollenkörner mediterraner Pflanzen (z.B. Olea) nördlich der Alpen gefunden werden. Während die Pollenanalyse die extralokale bis regionale Vegetation rekonstruieren kann, erlaubt die Analyse von Makrofossilien, das lokale Vorkommen einer Art zu bestimmen. Dies, da grössere Pflanzenteile (mit der Ausnahme flugfähiger Samen) nicht wie Pollen über weite Distanzen verbreitet werden. Stomata von Nadelhölzern sind ein willkommener Ersatz für Nadeln und werden bei der Pollenanalyse mitausgezählt (Trautmann 1953, Ammann et al 2014).

\section{Abwesenheitsproblem}

Das sogenannte Abwesenheitsproblem bleibt bis heute ungelöst: Die Abwesenheit von Mikro- oder Makrofossilien ist kein Hinweis auf die Abwesenheit einer Art an einem Standort. Der Grund dafür liegt darin, dass einzelne Individuen im Vergleich zur restlichen Vegetation viel zu wenig Pollen bzw. Makrofossilien produzieren, um nachgewiesen werden zu können. Aus diesem Grund ist es nach Welten
(1944; siehe auch Tinner \& Lotter 2006) unmöglich, die früheste Ankunft von einzelnen Individuen zu rekonstruieren. Jedoch können mit der Pollen- und der Makrorestanalyse die Anwesenheit von Populationen rekonstruiert werden. Da eine Mindestgrösse der Populationen nötig ist, um sie im Pollen- und Makrorestbild nachzuweisen, spricht man auch von spätester Ankunft (latest arrival) einer Art an einem Ort. Dies entspricht etwa der empirischen Grenze nach Lang (1994; zusammenhängende Pollenkurve, ca. 0.1-0.2\%). Für schwache Pollenproduzenten wie Tilia können einzelne Pollenkörner (absolute Pollengrenze) bereits das lokale Vorhandensein markieren. Dasselbe gilt für Makrorest- und Stomatavorkommen (Lang 1994). Aus der spätesten Ankunft einer Art an einem Ort kann die minimale Ausbreitungsgeschwindigkeit einer Population ermittelt werden. Dies ist von besonderem Interesse, um abzuschätzen, ob Baumarten fähig wären, mit einer zukünftigen Erwärmung des Klimas Schritt zu halten und in geeignete Breitengrade auszuweichen. Eine Tabelle zur groben Schätzung räumlicher Ausbreitungsgeschwindigkeiten findet sich in Tinner et al (2018). Die exponentielle Zunahme von Pollenprozenten, also das Erreichen der rationalen Pollengrenze, ist ein deutlicher Hinweis auf das Populationswachstum und die lokale Ausbreitung einer Art. Letztlich müssen für genaue Betrachtungen im Raum zahlreiche, gut datierte Pollendiagramme vorliegen.

\section{Nachweis von menschlichem Einfluss}

Die Jäger und Sammler der Alt- und Mittelsteinzeit hatten keinen nachweisbaren Einfluss auf die Vegetation. Mit dem Beginn des Ackerbaus in der Jungsteinzeit vor ca. 7500 Jahren setzten dagegen drei grundlegende Veränderungen ein: Rodungen (insbesondere Brandrodungen), der Anbau von Kulturpflanzen (insbesondere Getreide, siehe Cerealia in Abbildung 3b) und das Auftauchen bzw. die Zunahme von Kulturbegleitern (den «Unkräutern»). Da die tieferen Lagen im Früh-Holozän vollständig bewaldet waren, setzten die jungsteinzeitlichen Bauern Feuer ein, um Flächen für den Ackerbau zu gewinnen (Gobet et al 2010, Schwörer et al 2015, Rey et al 2018, Tinner et al 1999). Mit dem Anbau von Kulturpflanzen wurden auch Ackerbegleitpflanzen eingeschleppt (sogenannte Adventiten) und gewisse einheimische Pflanzen gefördert (sogenannte Apophyten). Das erstmalige Auftauchen von Adventiten wie Plantago lanceolata (Spitzwegerich) oder die starke Ausbreitung von Apophyten wie Alnus viridis (Grünerle) kann mittels Pollenanalyse ebenfalls nachgewiesen werden und ist ein eindeutiges Indiz für menschliche Landnutzung. Ähnlich verhält es sich mit Pilzsporen von Arten, die bevorzugt auf tierischem Dung wachsen (z.B. Sporormiella sp.). 


\section{Potenzial zur Zusammenarbeit mit Nachbardisziplinen}

Für die Archäologie ist die Bestimmung von Pollen und pflanzlichen Grossresten wichtig. So entstand schon sehr früh die Disziplin der Archäobotanik (z.B. Heer 1865, Jacomet \& Kreuz 1999). Interessant kann hierbei der Vergleich von Proben innerhalb und ausserhalb der Siedlung sein (on-site Archäobotanik versus off-site Paläoökologie). Sediment innerhalb der Siedlungen enthält oft Makrofossilien und Pollen von Nutzpflanzen und erlaubt deshalb Rückschlüsse auf die Ernährung und Lebensweise der Menschen. Sedimentproben ausserhalb von Siedlungen bilden die Vegetation der Umgebung ab und ermöglichen, den menschlichen Einfluss auf die Vegetation abzuschätzen.

Für die Genetik und die Phylogeografie entwickelte sich eine interessante Kooperation zwischen Paläoökologie und Forstgenetik: Mittels genetischer Methoden kann die Verwandtschaft verschiedener Populationen im Raum bestimmt werden, was Hypothesen zur Ausbreitung seit der letzten Eiszeit erlaubt. Die Paläoökologie kann dazu den Zeitpunkt der frühesten Anwesenheit von Populationen beitragen. Die Überlagerung von Karten des ersten (vermuteten) pollen- oder makrorestbasierten lokalen Auftretens mit phylogeografischen Karten kann so die zeitliche und räumliche Ausbreitung von Arten visualisieren (z.B. für Fagus Magri et al 2006, für die Alpen Gugerli \& Sperisen 2010). Mit neusten Sequenziermethoden ist es zudem möglich, die genetische Zusammensetzung vergangener Individuen und Populationen zu rekonstruieren, was erlaubt, den Einfluss von Störungen oder Klimaänderungen auf die genetische Vielfalt abzuschätzen (Schmid et al 2017).

Um dynamische (prozessbasierte) ökologische Modelle zu entwickeln, ist der Kontakt der Ökologie mit der Nachbardisziplin Paläoökologie essenziell. Denn nur mittels paläoökologischer Daten ist es möglich, die Modelle in der Zeit zu validieren (z.B. Henne et al 2015, Schwörer et al 2014). Mittels solcher Modelle (z.B. LANDCLIM, FORCLIM), können wertvolle Voraussagen zu künftigen Vegetationszuständen gemacht werden (Henne et al 2015, Schwörer et al 2014). Diese forstwirtschaftlich relevanten Erkenntnisse können allein aus der heutigen Ökologie oder der Verteilung der Arten im Raum, d.h. über statische oder korrelative Artenverteilungs- oder Bioklimanischenmodelle (SDM), nicht gewonnen werden (Henne et al 2015, Birks \& Tinner 2016).

Für die Klimaforschung gibt es verschiedene Anwendungen. Oft wurden paläobotanische Ergebnisse zur Rekonstruktion der Klimageschichte verwendet (z.B. Aarnes et al 2012, Birks \& Seppä 2004). Umgekehrt, wenn Klimaveränderungen aus unabhängigen Datensätzen vorliegen, zum Beispiel von Sauerstoffisotopen oder Chironomiden (Tabelle 1), lässt sich aus hoch aufgelösten Pollendiagrammen die Reaktion der Vegetation auf rasche Erwärmungen ablesen, was wichtig für die Abschätzung künftiger Klimafolgen ist (Tinner \& Lotter 2001, Ammann et al 2000, 2013).

\section{Vom Pollenprofil zur Vegetations- geschichte}

Durch die Pollen- und Makrorestanalyse sowie phylogenetische Studien ist bekannt, dass die meisten Baumarten in den südeuropäischen Halbinseln überdauerten. Da diese Regionen alle auch gebirgig sind, konnten sie dort in mittleren Höhenlagen überleben, wo es für sie dank Steigungsregen nicht zu trocken (wie im Tiefland) und nicht zu kalt (wie im Hochgebirge) war. Nebst diesen südlichen Refugien wurden aber auch kleinere Refugialgebiete am Alpensüdrand oder in Osteuropa gefunden. Die Wiederbesiedlung durch die wichtigsten Baumarten der Schweiz nach der letzten Eiszeit wurde kürzlich hier durch Tinner et al (2018) dargestellt: Das der Schweiz am nächsten liegende, gesicherte Refugium temperierter Arten (z.B. Linden, Buche, Eschen, Ulmen, Tanne, Eichen) befand sich in den Colli Euganei, nur etwas mehr als $200 \mathrm{~km}$ vom Tessin entfernt.

Das Spätglazial war geprägt von markanten und zum Teil rasanten Vegetationsveränderungen, die am Beispiel des Lobsigensees illustriert werden können (Abbildung 3a): Für die Älteste Dryas sind Gattungen und Arten dokumentiert, wie sie heute als Pioniere in Gletschervorfeldern, aber auch in Steppen gedeihen. Gehölze beschränkten sich weitgehend auf Zwergbirken und verschiedene Zwergweiden. Mit der raschen Erwärmung am Übergang von Ältester Dryas zum Bölling (also dem Beginn des sog. spätglazialen Interstadials) vor ca. $14700 \mathrm{Jah}$ ren breiteten sich zum ersten Mal nach der letzten Eiszeit wieder Waldgesellschaften im Mittelland aus. Zwergbirken wurden durch Baumbirken ersetzt, und die Populationen von Hippophaë und Juniperus nahmen schnell zu. Das Alleröd als zweiter Teil des spätglazialen Interstadials war dann von Pinus dominiert. Der Kälterückschlag der Jüngeren Dryas (ca. $-4{ }^{\circ} \mathrm{C}$ ) öffnete im Mittelland auf 500 m ü.M. die Wälder vor ca. 12500 Jahren nur leicht.

Das Holozän (die jetzige Warmzeit) begann vor ca. 11700 Kalenderjahren mit Veränderungen von grosser Amplitude und Geschwindigkeit (Abbildung 3b); Hauptursache war eine rasante Erwärmung des Klimas von etwa zwei bis vier Grad Celsius innerhalb weniger Jahrzehnte. Die von Corylus dominierten Mischwälder des Früh-Holozäns wie auch der anschliessende «Eichenmischwald» aus Ulmus, Quercus, Tilia und Fraxinus haben in der Gegenwart kaum ein Analogon in der Schweiz. Mit der Jungsteinzeit führte erstmals der Mensch zu Vegetations- 
veränderungen. Als Beispiele mögen die CerealiaFunde ab ca. 4000 v. Chr. und die Juglans-Funde ab der Römerzeit dienen (Abbildung 3b). Im Mittelalter fällt der markante Gipfel von Cannabis im Lobsigensee auf - der kleine See wurde wohl als Rösteplatz verwendet, denn zur Loslösung der Fasern für die Herstellung von Seilen und Textilien mussten die Hanfstengel zuerst im Wasser des Sees eingeweicht werden. Paläoökologische Datenbanken speichern die vielen im Laufe der Zeit gewonnen Daten, zum Beispiel die European Pollen Database ${ }^{1}$ oder die Neotoma Paleoecology Database ${ }^{2}$. Eine gute Übersicht zu Vegetationsänderungen seit der letzten Eiszeit findet sich auch in Gobet et al (2010) und Lang (1994).

\section{Schlussfolgerungen}

Die Paläoökologie erlaubt einen ganzheitlichen Blick auf Vegetationsänderungen über lange Zeiträume und ist daher ein wichtiges Werkzeug für die Identifikation der natürlichen Vegetation und die Abschätzung zukünftiger Entwicklungen. Mittels paläoökologischer Methoden können Vegetationsprozesse untersucht werden, die über die Dauer eines Forschungsprojektes oder einer Langzeitversuchsfläche hinausgehen. Die Paläoökologie ist mittlerweile mehr als nur eine beschreibende Wissenschaft. Dank quantitativer Methoden wird sie auch verwendet, um ökologische Hypothesen zu testen und kausale Zusammenhänge herzustellen. Für die heutige Forstwirtschaft kann der Blick in die Vergangenheit besonders bei zwei Punkten von Interesse sein: 1) Wie die heutigen Wälder durch menschlichen Einfluss während Jahrtausenden geprägt wurden und 2) wie die verschiedenen Baumarten auf leicht trockenere und wärmere Klimabedingungen reagierten und reagieren werden. Mithilfe der paläoökologischen Zeitmaschine können wir also sowohl in die Vergangenheit reisen, als auch einen Blick in die Zukunft wagen.

Eingereicht: 31. August 2018, akzeptiert (mit Review): 26. Februar 2019

1 www.europeanpollendatabase.net (22.2.2019)

2 www.neotomadb.org (22.2.2019)

\section{Literatur}

AARNES I, KÜHL N, BIRKS HH (2012) Quantitative climate reconstruction from late-glacial and early Holocene plant macrofossils in western Norway using the probability density function approach. Rev Palaeobot Palynol 170: 27-39.

AMMANN B (2000) Biotic responses to rapid climatic changes: Introduction to a multidisciplinary study of the Younger Dryas and minor oscillations on an altitudinal transect in the Swiss Alps. Palaeogeogr Palaeoclimatol Palaeoecol 159: 191-201.
AMMANN B, VAN DER KNAAP WO, LANG G, GAILLARD MJ, KALTENRIEDER P ET AL (2014) The potential of stomata analysis in conifers to estimate presence of conifer trees: examples from the Alps. Veg Hist Archaeobot 23: 249-264.

AMMANN B, VAN RADEN UJ, SCHWANDER J, EICHER U, GILLI A ET AL (2013) Responses to rapid warming at Termination 1a at Gerzensee (Central Europe): Primary succession, albedo, soils, lake development, and ecological interactions. Palaeogeogr Palaeoclimatol Palaeoecol 391, Part B: 111-131.

BEUG HJ (2004) Leitfaden der Pollenbestimmung für Mitteleuropa und angrenzende Gebiete. München: Pfeil. 542 p.

BIRKS HJB, BIRKS HH (2016) How have studies of ancient DNA from sediments contributed to the reconstruction of Quaternary floras? New Phytol 209: 499-506.

BIRKS HJB, SEPPÄ H (2004) Pollen-based reconstructions of lateQuaternary climate in Europe - progress, problems, and pitfalls. Acta Palaeobot 44: 317-334.

BIRKS HJB, TINNER W (2016) European tree dynamics and invasions during the Quaternary. In: Krumm F, Vìtkovà L, editors. Introduced tree species in European forests: opportunities and challenges. Freiburg i.Br.: European Forest Institute. pp. 22-43.

BIRKS HJB, LOTTER AF, JUGGINS S, SMOL JP, EDITORS (2012) Tracking environmental change using lake sediments. Volume 5: Data handling and numerical techniques. Dordrecht: Springer. $745 \mathrm{p}$.

CH2018 (2018) Climate scenarios for Switzerland. Technical report. Zurich: National Centre for Climate Services. 271 p.

CONEDERA M, TINNER W (2010) Langzeit-Feuerökologie der Schweiz. Schweiz Z Forstwes 161: 424-432. doi: 10.3188/ szf.2010.0424

CONEDERA M, TINNER W, CRAMERI S, TORRIANI D, HEROLD A (2006) Taxon-related pollen source areas for lake basins in the southern Alps: an empirical approach. Veg Hist Archaeobot 15: 263-272.

DAVIS OK, SHAFER DS (2006) Sporormiella fungal spores, a palynological means of detecting herbivore density. Palaeogeogr Palaeoclimatol Palaeoecol 237: 40-50.

EGGERMONT H, HEIRI O (2012) The chironomid-temperature relationship: expression in nature and palaeoenvironmental implications. Biol Rev 87: 430-456.

ELLENBERG H, LEUSCHNER C (2010) Vegetation Mitteleuropas mit den Alpen in ökologischer, dynamischer und historischer Sicht. Stuttgart: Ulmer, 6 ed. 1333 p.

FINSINGER W, SCHWÖRER C, HEIRI O, MORALES-MOLINO C, RIBOLINI A ET AL (2018) Fire on ice and frozen trees? Inappropriate radiocarbon dating leads to unrealistic reconstructions. New Phytol. doi: 10.1111/nph.15354

GOBET E, VESCOVI E, TINNER W (2010) Ein paläoökologischer Beitrag zum besseren Verständnis der natürlichen Vegetation der Schweiz. Bot Helv 120: 105-115.

GUGERLI F, PARDUCCI L, PETIT RJ (2005) Ancient plant DNA: Review and prospects. New Phytol 166: 409-418.

GUGERLI F, SPERISEN C (2010) Genetische Struktur von Waldbäumen im Alpenraum als Folge (post)glazialer Populationsgeschichte. Schweiz Z Forstwes 161: 207-215. doi: 10.3188/ szf. 2010.0207

HAFNER A, SCHWÖRER C (2018) Vertical mobility around the highalpine Schnidejoch Pass. Indications of Neolithic and Bronze Age pastoralism in the Swiss Alps from paleoecological and archaeological sources. Quat Int 484: 3-18.

HEER O (1865) Die Pflanzen der Pfahlbauten. Neujahrsbl Nat. forsch Ges Zür 68: 1-54.

HEIRI O, KOINIG KA, SPÖTL C, BARRETT S, BRAUER A ET AL (2014) Palaeoclimate records $60-8 \mathrm{ka}$ in the Austrian and Swiss Alps and their forelands. Quat Sci Rev 106: 186-205.

HEIRI O, LOTTER AF, LEMCKE G (2001) Loss on ignition as a method for estimating organic and carbonate content in sediments: reproducibility and comparability of results. J Paleolimnol 25: 101-110. 
HENNE PD, ELKIN C, FRANKE J, COLOMBAROLI D, CALÒ C ET AL (2015) Reviving extinct Mediterranean forest communities may improve ecosystem potential in a warmer future. Front Ecol Environ 13: 356-362.

JACOMET S, KREUZ A (1999) Archäobotanik. Stuttgart: Ulmer. $368 \mathrm{p}$.

LANG G (1994) Quartäre Vegetationsgeschichte Europas. Jena: Fischer. 462 p.

LAST WM, SMOL JP, EDITORS (2001A) Tracking environmental change using lake sediments. Volume 2: Physical and geochemical methods. Dordrecht: Kluwer. 502 p.

LAST WM, SMOL JP, EDITORS (2001B) Tracking environmental change using lake sediments. Volume 1: Basin analysis, coring, and chronological techniques. Dordrecht: Kluwer. 548 p.

MAGNY M (2004) Holocene climate variability as reflected by mid-European lake-level fluctuations and its probable impact on prehistoric human settlements: The record of human/ climate interaction in lake sediments. Quat Int 113: 65-79.

MAGRI D, VENDRAMIN GG, COMPS B, DUPANLOUP I, GEBUREK T ET AL (2006) A new scenario for the Quaternary history of European beech populations: palaeobotanical evidence and genetic consequences. New Phytol 171: 199-221.

REY F, GOBET E, SCHWÖRER C, WEY O, HAFNER A ET AL (2018) Causes and mechanisms of synchronous succession trajectories in primeval Central European mixed Fagus sylvatica forests. J Ecol. doi: 10.1111/1365-2745.13121

SCHMID S, GENEVEST R, GOBET E, SUCHAN T, SPERISEN C ET AL (2017) HyRAD-X, a versatile method combining exome capture and RAD sequencing to extract genomic information from ancient DNA. Methods Ecol Evol 8: 1374-1388.

SCHWÖRER C, COLOMBAROLI D, KALTENRIEDER P, REY F, TINNER W (2015) Early human impact (5000-3000 BC) affects mountain forest dynamics in the Alps. J Ecol 103: 281-295.

SCHWÖRER C, HENNE PD, TINNER W (2014) A model-data comparison of Holocene timberline changes in the Swiss Alps reveals past and future drivers of mountain forest dynamics. Glob Chang Biol 20: 1512-1526.

\section{Les forêts dans une machine à voyager dans le temps - possibilités et limites de la paléoécologie}

La paléoécologie permet de reconstituer des processus écologiques qui se déroulent sur de très longues périodes - par exemple la dynamique de la végétation depuis la dernière période glaciaire ou les changements dans la composition de la forêt suite à l'établissement humain. On y parvient en analysante des restes végétaux tels que du pollen, des spores, des feuilles, des graines ou du charbon de bois, qui ont été conservés dans les sédiments des lacs et des tourbières pendant des milliers d'années en l'absence d'air. L'article présente les principes, les limites et le potentiel de la paléoécologie. II devient clair que cette discipline est beaucoup plus qu'une science descriptive aujourd'hui. Grâce à des méthodes quantitatives, elle est maintenant aussi utilisée pour tester des hypothèses écologiques et établir des relations de cause à effet. La paléoécologie peut donc aider à évaluer l'adaptabilité ou la résilience des communautés végétales aux perturbations externes, et fournit ainsi des informations importantes sur la réponse de nos forêts au changement climatique passé, actuel et futur.
SMOL JP, BIRKS HJB, LAST WM, EDITORS (2001A) Tracking environmental change using lake sediments. Volume 3: Terrestrial, algal and siliceous indicators. Dordrecht: Kluwer. $371 \mathrm{p}$.

SMOL JP, BIRKS HJB, LAST WM, EDITORS (2001B) Tracking environmental change using lake sediments. Volume 4: Zoological indicators. Dordrecht: Kluwer. 271 p.

SUGITA S (2007A) Theory of quantitative reconstruction of vegetation II: all you need is LOVE. Holocene 17: 243-257.

SUGITA S (2007B) Theory of quantitative reconstruction of vegetation I: pollen from large sites reveals regional vegetation composition. Holocene 17: 229-241.

TINNER W, HUBSCHMID P, WEHRLI M, AMMANN B, CONEDERA M (1999) Long-term forest fire ecology and dynamics in southern Switzerland. J Ecol 87: 273-289.

TINNER W, LOTTER AF (2001) Central European vegetation response to abrupt climate change at $8.2 \mathrm{ka}$. Geology 29: $551-$ 554.

TINNER W, LOTTER AF (2006) Holocene expansions of Fagus silvatica and Abies alba in Central Europe: where are we after eight decades of debate? Quat Sci Rev 25: 526-549.

TINNER W, VAN DER KNAAP W, CONEDERA M, AMMANN B (2018) Invasionen und Zusammenbrüche von Baumarten nach der Eiszeit. Schweiz Z Forstwes 169: 60-68. doi: 10.3188/szf.2018.0060 TRAUTMANN W (1953) Zur Unterscheidung fossiler Spaltöffnungen der mitteleuropäischen Coniferen. Flora 140: 523-533.

VAN DER KNAAP W, VAN LEEUWEN J, SVITAVSKÁ-SVOBODOVÁ H, PIDEK I, KVAVADZE E ET AL (2010) Annual pollen traps reveal the complexity of climatic control on pollen productivity in Europe and the Caucasus. Veg Hist Archaeobot 19: 285-307.

WELTEN M (1944) Pollenanalytische, stratigraphische und geochronologische Untersuchungen aus dem Faulenseemoos bei Spiez. Bern: Huber, Veröff Geobot Inst Rübel 21. 201 p.

WELTEN M (1950) Beobachtungen über den rezenten Pollenniederschlag in alpiner Vegetation. In: Rübel W, Lüdi W, editors. Bericht über das geobotanische Forschungsinstitut Rübel in Zürich für das Jahr 1949. Zürich: Buchdruckerei Berichthaus. pp. $48-57$.

\section{Forests in a time machine - possibilities and limits of paleoecology}

Paleoecology allows the reconstruction of ecological processes that take place on long timescales - for example the vegetation dynamics since the last Ice Age or changes in the species composition of forests due to anthropogenic land use. This can be achieved by analyzing plant remains like pollen, spores, leaves, seeds or charcoal that have been conserved over millennia under low oxygen conditions in the sediment of lakes and mires. In this article, we outline the principals, the limits and the potential of paleoecology. It becomes clear that nowadays, this discipline is more than just a descriptive science. Thanks to quantitative methods, it can be used to test ecological hypothesis and identify causal relationships. Paleoecology can therefore contribute to assess the resilience of vegetation communities to external disturbances, and provides important information on how our forests will react to past, current and future climate change. 